\title{
SISTEMAS LINEALES DINÁMICOS INVARIANTES EN SERIES CRONOLÓGICAS, CON APLICACIONES A ESTUDIOS ACUÁTICOS y Climatológicos del Perú
}

Fátima Medina Merino de Aliaga

\section{Resumen}

En el presente artículo se desarrollan los procesos que generan las series de tiempo como sistemas lineales dinámicos invariantes, con una aplicación al modelamiento del desembarque de anchoveta en el litoral peruano como serie de entrada y la temperatura superficial del mar como serie de salida, para lo cual se hace uso de la función de transferencia. Además, se realiza la aplicación de la coherencia espectral para medir el grado de asociación entre las series del sistema.

Palabras clave: sistemas lineales / función de transferencia / coherencia espectral

IחדーRーA 


\section{Introducción}

En el trabajo con series cronológicas, la idea de realizar estimaciones a futuro, detectar factores no observables que afectan las estimaciones y muestran situaciones inusitadas resulta de gran interés. Sin embargo, pocas veces se trabaja el modelamiento de series cronológicas (o series de tiempo) como un sistema. El objetivo de este artículo es mostrar los procesos que generan las series cronológicas como sistemas lineales dinámicos invariantes, presentando algunas situaciones relacionadas con el tema ambiental y climático, el cual actualmente es de gran interés general. Asimismo, se ha puesto especial énfasis en los procesos bivariados.

Otro de los objetivos es el de mostrar la función de transferencia como herramienta fundamental para el problema de series de tiempo bivariadas; para lo cual se presentan los conceptos básicos necesarios para la definición del sistema, su comportamiento y caracterización para modelos continuos.

Al presentar esta gama de sistemas para series cronológicas se alentará el uso de modelos de predicción diversos, innovadores y alternativos a los utilizados tradicionalmente. Pero principalmente, este trabajo constituye un llamado a los expertos en análisis y programación de sistemas para que apliquen sus conocimientos en desarrollar los aplicativos que se requieren.

\section{Sistemas lineales}

\subsection{Procesos estocásticos univariados y bivariados}

Inicialmente se presentarán las definiciones básicas que son utilizadas en este trabajo.

- Definición 1.1. Se denomina proceso aleatorio $\left\{X_{t(t)}\right\}$ a una familia de variables aleatorias, cuando $t$ pertenece a un conjunto ordenado en el tiempo $T$.

- Definición 1.2. Se dice que $\left\{X_{(t)}\right\}$ es un proceso de parámetros continuos si t toma valores reales en un intervalo de tiempo continuo (finito o infinito), tal que Tí $R^{1}\left\{X_{(t)}\right\}$.

- Definición 1.3. Se dice que $\left\{X_{\}}\right\}$es un proceso de parámetros discretos, si t toma un conjunto de valores discretos $t=0, \pm 1, \pm 2, \ldots$

- Definición 1.4. Se denomina realización de un proceso aleatorio $\left\{X_{(t)}\right\}$ al valor $X_{(t)}$ que toma la variable aleatoria. Es decir, sea una variable aleatoria $X_{(t)^{\prime}} \forall t$ de tal manera que cada vez que se hace una idealización de los valores de $X_{(t)}$ en el experimento se tiene un proceso aleatorio, cuando se concretiza el experimento se obtiene una realización del proceso. Por tanto, para el caso discreto, una realización del proceso estocástico $\left\{X_{t}\right\}$ es uno de la serie de valores $\left(\ldots, X_{-2^{\prime}} X_{-1^{\prime}}\right.$ 
$\left.X_{0^{\prime}} X_{1}, \ldots\right)$ a los que puede dar lugar. La realización puede ser considerada como un miembro del proceso del mismo modo en que una observación individual es considerada como el miembro de una población (Box, Jenkins y Reinsel 1994).

- Ejemplo 1. Sea $X_{t}$ una variable aleatoria con función de distribución Bernoulli, para $t=1,2, \ldots, 10$. Una posible realización del proceso será: 0, 1, 0, 1, 1, 1, 1, 0 , $1,0$. Y de esta manera podría tenerse $2^{10}$ distintas realizaciones a la totalidad de las cuales se denominará ensamble.

Sin embargo, cuando t representa ubicación temporal real, no es posible tener varias realizaciones de un proceso.

- Ejemplo 2. Sea $X_{(t)}$ la temperatura superficial del mar peruano en el instante t. Si t es el tiempo real, es evidente que se tendrá una sola realización, pues cada instante tocurre una sola vez y no existen repeticiones.

\subsection{Sistemas lineales de causa efecto}

- Definición 2.1. El proceso $\left\{X_{(t)}\right\}$ recibe el nombre de proceso de entrada si explica el comportamiento de otro proceso $\left\{Y_{(t)}\right\}$ llamado proceso de salida.

- Definición 2.2. El modelo formado por dos procesos univariados, entrada y salida, relacionados a través de un filtro, recibe el nombre de sistema.

- Definición 2.3. Un sistema dinámico se denomina sistema dinámico lineal si el filtro que relaciona los procesos de entrada y de salida es de tipo lineal.

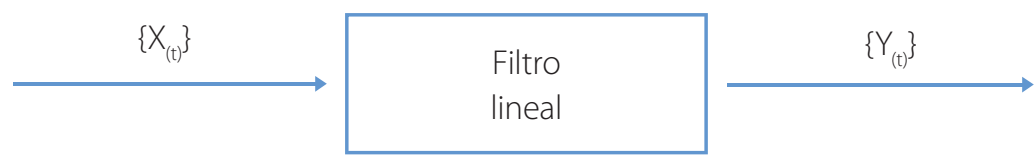

Figura 1. Sistema dinámico lineal

Elaboración propia.

Luego los siguientes sistemas son ejemplos de sistemas dinámicos lineales:
a) $Y_{t}=(1 / 2)\left[X_{t-2}+X_{t-1}+X_{t}+X_{t+1}+X_{t+2}\right]$
b) $Y_{t}=X_{t}-X_{t-1}$
c) $Y_{t}=P_{0}+P_{1} X_{(t-1)}+P_{2} X_{(t-2)}+\ldots+P_{b} X_{(t-b)}$ 
- Definición 2.4. Se denomina función impulso respuesta a la función de ponderaciones que describe la forma en que el proceso de salida se relaciona con el proceso de entrada del sistema dinámico lineal. Sea I l( la función impulso respuesta para procesos en tiempos continuos e $\mathrm{I}_{\mathrm{h}}$ en tiempos discretos.

- Definición 2.5. Dados $\left\{X_{(t)}\right\}$ e $\left\{Y_{(t)}\right\}$, forman un sistema dinámico lineal invariante en el tiempo, si para $h$ períodos de retardo, $\left\{X_{(t-h)}\right\}$ produce $\left\{Y_{(t-h)}\right\}$. De esta manera, el valor de $h$ (períodos de retardo) para los casos (a), (b) y (c) del ejemplo 1, es de la siguiente forma:

a) Para $t-2, t-1, t, t+1, t+2$ se tiene $h=2,1,0,-1,-2$, respectivamente.

b) Para $t, t-1$ se tiene que $h=0,1$, respectivamente.

c) Para (t-1), (t-2), .., (t-b) se tiene que $h=1,2, \ldots, b$, respectivamente.

- Definición 2.6. Para dos procesos $\left\{X_{(t)}\right\}$ e $\left\{Y_{(t)}\right\}$ relacionados en tiempos continuos, es decir $-\infty<\mathrm{t}<\infty$, se define un sistema dinámico lineal invariante en $\mathrm{t}$ como:

$$
\mathrm{Y}_{(\mathrm{t})}=\int_{-\infty}^{\infty} \mathrm{I}_{(\mathrm{h})} \mathrm{X}_{(\mathrm{t}-\mathrm{h})} \mathrm{dh}
$$

- Definición 2.7. Sean dos procesos $\left\{X_{t}\right\}$ e $\left\{Y_{t}\right\}$ relacionados en tiempos discretos, se define un sistema lineal dinámico invariante (SLDI) en t como:

$$
\mathrm{Y}_{\mathrm{t}}=\sum_{\mathrm{h}=-\infty}^{\infty} \mathrm{I}_{\mathrm{h}} \mathrm{X}_{\mathrm{t}-\mathrm{h}}
$$

Otra expresión para un SLDI con procesos $\left\{X_{\mathrm{r}}\right\}$ e $\{Y\}$ que lo conforman y que es una consecuencia de la definición 2.7 es:

$Y_{t}=\left(I_{0}+I_{1} B+I_{2} B^{2}+\ldots\right) X_{t-h}$, donde $B$ es un operador de retardo, $Y_{t}=I(B) X_{t-h,}{ }^{\prime \prime} h^{3} 0$.

Al trabajar con sistemas lineales dinámicos invariantes en el tiempo, se está asegurando que la función impulso respuesta no depende del tiempo t.

A continuación se presentan dos situaciones, con relación al tiempo que demora el proceso de entrada en ajustar el proceso de salida, en un sistema dinámico:

a) El proceso de entrada $\left\{X_{(t)}\right\}$ influye en el proceso de salida $\left\{Y_{(t)}\right\}$ en el mismo momento t. Esto es, por cada observación en la variable de entrada se obtiene una respuesta en la salida. 
Ejemplo:

La temperatura de la superficie oceánica en el momento $t_{1}$ influye en el grado de absorción de dióxido de carbono en el mismo t (Vidal 1997).

b) $\left\{X_{(t)}\right\}$ no influye de forma inmediata en $\left\{Y_{(t)}\right\}$, pero sí produce una respuesta en $\left\{Y_{(t+h)}\right\}$, o dicho de otra forma: $\left\{X_{(t-h)}\right\}$ influye en $\left\{Y_{(t)}\right\}$ mediante una relación que se denominará relación de retardo.

Ejemplo:

El aumento de la temperatura superficial del mar peruano en t produce una respuesta en la biomasa de anchoveta $\mathrm{t}+\mathrm{h}$ momentos en el tiempo, ya que un cambio en la temperatura superficial afecta el desove de las especies pelágicas (Ñiquen y Bouchon 2004).

\subsection{Función de transferencia}

- Definición 3.1. La función de transferencia $f_{(t)}$ se define como la función característica de la función impulso respuesta $\left.\right|_{(h)^{\prime}}$ así para procesos en tiempo continuo:

$$
\phi_{(\mathrm{t})}=\int_{0}^{\infty} \mathrm{e}^{\mathrm{itu}} \mathrm{I}_{(\mathrm{u})}=\mathrm{du}, \quad 0<\mathrm{t}<\infty
$$

Análogamente para procesos en tiempos discretos:

$$
\phi_{\mathrm{t}}=\sum_{\mathrm{u}=0}^{\infty} \mathrm{e}^{\mathrm{itu}} \mathrm{I}_{(\mathrm{u})}, \quad 0<\mathrm{t}<\pi
$$

Antes de introducir la definición de la función respuesta de paso del sistema lineal dinámico, se presenta la siguiente figura para el proceso de entrada:

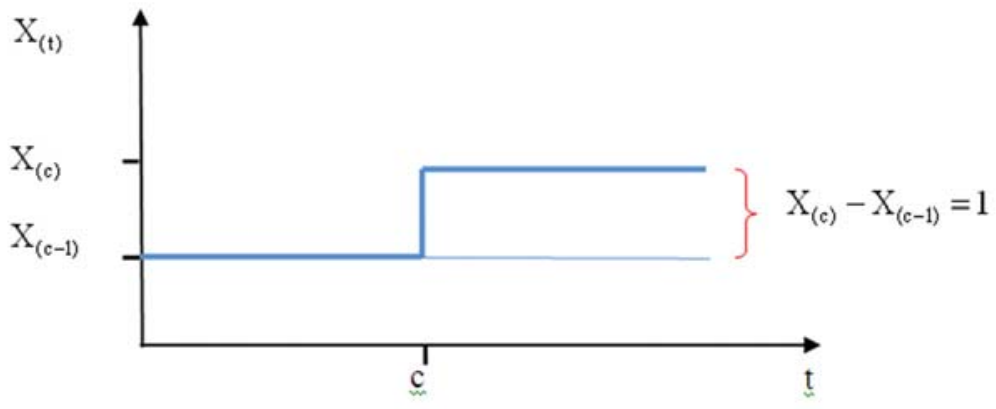

Figura 2. Respuesta de paso del sistema lineal dinámico

Elaboración propia. 
- Definición 3.2. La función respuesta de paso se define como la función que describe la respuesta del sistema cuando en el proceso de entrada se produce un salto a partir de un tiempo $\mathrm{c} \in\langle-\mathrm{t}, \mathrm{t}\rangle$. Si los procesos son dados para tiempos continuos, la respuesta de paso se define como:

$$
\mathrm{P}_{(\mathrm{t})}=\int_{-\infty}^{\mathrm{t}} \mathrm{I}_{(\mathrm{h})} \mathrm{dh}
$$

Cuando se tiene un salto en el proceso de entrada, el cual produce una función respuesta de paso, es lógico pensar que en el proceso de salida también se presentará un cambio, relacionado con la respuesta de paso (Priestley 1981). Entonces, se define:

- Definición 3.3. La ganancia (g) en el SLDI se define como una cantidad constante que representa el cambio en $\left\{Y_{\mathrm{r}}\right\}$ cuando en $\left\{X_{\mathrm{r}}\right\}$ se presenta un salto de una unidad (respuesta de paso en el sistema lineal dinámico invariante). En el caso de aplicarse una función respuesta de paso al SLDI, la función impulso respuesta se convierte en la ganancia del sistema (g) porque toma un valor constante.

Ejemplo 1:

$$
\text { Sea } \mathrm{Y}_{\mathrm{t}}=\sum_{\mathrm{h}=-\infty}^{\infty} \mathrm{I}_{\mathrm{h}} \mathrm{X}_{\mathrm{t}-\mathrm{h}} \text {, si } \mathrm{h}=0 \text { entonces } \mathrm{Y}_{\mathrm{t}}=\mathrm{gX} \text {. }
$$

Ejemplo 2:

Sea $\left\{X_{(t)}\right\}$ Precipitaciones pluviales e $\left\{Y_{(t)}\right\}$ Caudal medio del río $X$. Para este ejemplo se observa que el volumen de las precipitaciones pluviales genera un aumento o disminución, de manera inmediata, del caudal del río $X$, y en consecuencia el valor de $h$ (períodos de retardo) es cero. Luego, en $Y_{t}=I_{0} X_{t}$ el valor $I_{o^{\prime}}$ que en realidad para este caso es el único coeficiente de la función, nos dice en cuánto se incrementa el caudal medio del río X por cada unidad de cambio en las precipitaciones pluviales, $\mathrm{I}_{0}=\mathrm{g}$ recibe el nombre de ganancia del proceso en el momento t.

Como en el caso de series de tiempo univariadas puede ser conveniente trabajar con las diferencias finitas, por ejemplo para conseguir que sean estacionarias, surge entonces la necesidad de saber qué ocurre con la función de transferencia si se aplican las diferencias finitas.

- Lema 3.1. Las diferencias finitas de los procesos de entrada y salida, en tiempos discretos, con respecto a su propio pasado, satisfacen la misma función de transferencia definida para procesos originales.

Demostración:

Sean:

$y_{t}=Y_{t}-Y_{t-1}$ 
$\mathrm{x}_{\mathrm{t}}=\mathrm{X}_{\mathrm{t}}-\mathrm{X}_{\mathrm{t}-1}$

Entonces, por definición 2.7

$Y_{t}=I_{0} X_{t}+I_{1} X_{t-1}+I_{2} X_{t-2}+\ldots$

$Y_{t-1}=I_{0} X_{t-1}+I_{1} X_{t-2}+I_{2} X_{t-3}+\ldots$

Luego:

$Y_{t}-Y_{t-1}=I_{0}\left(X_{t}-X_{t-1}\right)+I_{1}\left(X_{t-1}-X_{t-2}\right)+\ldots$

Siendo $\mathrm{y}_{\mathrm{t}}=\mathrm{Y}_{\mathrm{t}}-\mathrm{Y}_{\mathrm{t}-1} ; \mathrm{X}_{\mathrm{t}}=\mathrm{X}_{\mathrm{t}}-\mathrm{X}_{\mathrm{t}-1} ; \ldots ; \mathrm{X}_{\mathrm{t}-1}=\mathrm{X}_{\mathrm{t}-1}-\mathrm{X}_{\mathrm{t}-2} ; \mathrm{X}_{\mathrm{t}-2}=\mathrm{X}_{\mathrm{t}-2}-\mathrm{X}_{\mathrm{t}-3} ; \ldots$

$\mathrm{y}_{\mathrm{t}}=\mathrm{I}_{0} \mathrm{x}_{\mathrm{t}}+\mathrm{I}_{1} \mathrm{x}_{\mathrm{t}-1}+\mathrm{I}_{2} \mathrm{x}_{\mathrm{t}-2}+\ldots$

$y_{t}=\left(I_{0}+I_{1} B+\ldots\right) x_{t}$

$\mathrm{y}_{\mathrm{t}}=\mathrm{I}(\mathrm{B}) \mathrm{x}_{\mathrm{t}}$

- Lema 3.2. En el caso de SLDI generalizados expresados como ecuaciones diferenciales lineales con coeficientes en tiempos continuos T.DY ${ }_{(t)}+Y_{(t)}$ $=g X_{(t)^{\prime}}$ donde $T$ es una constante y $\mathrm{PY}_{(\mathrm{t})}=\partial \mathrm{Y}_{(\mathrm{t})} / \partial \mathrm{t}$ estos satisfacen la definición de función de transferencia dada en la definición 3.1.

\subsection{Relación entre los procesos continuos y los procesos discretos}

Sean los procesos $\left\{X_{(t)}\right\}$ e $\left\{Y_{(t)}\right\}$ tomados en tiempos continuos, existe una forma de aproximarlos de manera general a procesos en tiempos discretos, y es mediante el método de entradas pulsadas. Para ello, sea el tiempo de longitud K en el que se registra el comportamiento del modelo, el cual se divide en $\mathrm{N}$ intervalos equiespaciados, y se observan los valores de la entrada en cada subunidad de tiempo [( $\left.\mathrm{k}_{\mathrm{i}} / \mathrm{N}\right]$ $=b, \forall i=0,1, \ldots$ que producirán sus respectivos valores de salida.

\begin{tabular}{cccccc}
\hline & 1 & & & & \\
$b+0$ & $b+1$ & $b+2$ & $b+3$ & $b+4$ & $\cdots$
\end{tabular}

Figura 3. Cambio de tiempos continuos a tiempos discretos por entradas pulsadas

Elaboración propia.

Naturalmente, a lo largo del intervalo $\langle b, b+1\rangle$, las observaciones a la variable de entrada se mantienen fijas en ese nivel. Posteriormente, para una entrada pulsada existe un filtro de tipo discreto que hace que $Y_{(t)}$ sea igual a $Y_{t^{\prime}}$ es decir, salidas en tiempos discretos (Box et al. 1994). 
- Lema 4.1. La relación de entrada-salida para procesos discretos en el tiempo $\left\{X_{(t)}\right\}$ e $\left\{Y_{(t)}\right\}$ es similar al que se establece para procesos continuos en el tiempo $\{X\}$ e $\{Y\}$, que forman un sistema lineal dinámico invariante en el tiempo, en términos de la función impulso respuesta.

Demostración:

Para $\left\{X_{(t)}\right\}$ e $\left\{Y_{(t)}\right\}$ se tiene, por la definición 2.6

$$
Y_{(t)}=\int_{0}^{\infty} I_{(h)} X_{(t-h)} d h
$$

Luego, el intervalo $[0, \infty\rangle$ puede dividirse así: $[b, b+1\rangle,[b+1, b+2\rangle,[b+2, b+3\rangle$,

$\ldots \forall b \geq 0$

De forma que:

$Y_{(t)}=\left[\int_{0}^{b+1} I_{(b+1)} d h\right] X_{[t-(b+1)]}+\left[\int_{b+1}^{b+2} I_{(b+2)} d h\right] X_{[t-(b+2)]}+\ldots$

$Y_{(t)}=\sum_{i=0}^{\infty} I_{b+i} X_{t-(b+i)}$

$Y_{(t)} \cong Y_{t}$

Por este resultado se concluye que los tratamientos empleados para procesos en tiempo continuo tienen también su equivalencia en procesos en tiempos discretos.

\subsection{Modelos de función de transferencia de primer orden}

Según las definiciones 2.6 y 2.7 y los lemas 3.1 y 3.2, el proceso de salida está en función del proceso de entrada retardada, así:

$$
\begin{array}{ll}
(1+T D) Y_{(t)}=g X_{(t-h)} & \text { por lema 3.1, o equivalentemente: } \\
(1+T D) Y_{t}=g X_{t-h} & \text { en procesos de tiempos discretos }
\end{array}
$$

En ambos casos es claro que se tienen ecuaciones diferenciales de primer orden. Si se trabaja un SLDI en el tiempo que comprende tres procesos, dos procesos de entrada y uno de salida, crecerá el orden de la ecuación diferencial que caracteriza el sistema. Así se tendrá una ecuación diferencial de segundo orden. Tal como se muestra a continuación: 


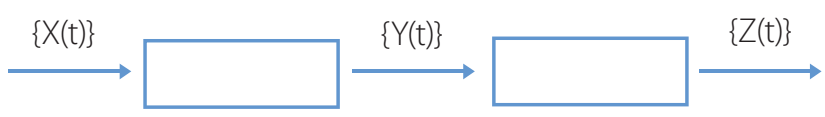

Figura 4. Modelo de función de transferencia de primer orden

Elaboración propia.

En el sistema que se muestra en la figura 4, se cumple:
$(1+T D) Y_{(t)}=g X_{(t-h)}$
(1) T constante
$\left(1+T_{1} D\right) Z_{(t)}=g_{1} Y_{(t)}$
(2) T1constante

Reemplazando la ecuación (1) en (2)

$\left(1+T_{1} D\right) Z_{(t)}=g_{1}\left[g \frac{X_{(t-h)}}{(1+T D)}\right]$

$\left(1+T_{1} D\right)(1+T D) Z_{(t)}=g_{1} g X_{(t-h)}$

Sea $g_{1} g=G$

$\left\{1+\left(T+T_{1}\right) D+\left(T_{1} T\right) D^{2}\right\} Z_{(t)}=G X_{(t-h)}$

Para $T+\mathrm{T}_{1}=\mathrm{C}_{1} \mathrm{y} \mathrm{T}_{1} \mathrm{~T}=\mathrm{C}_{2}$ constantes

$\left\{1+C_{1} D+C_{2} D^{2}\right\} Z_{(t)}=G X_{(t-h)}$

para $\mathrm{H}$ constante:

$\left\{1+C_{1} D+C_{2} D^{2}\right\} Z_{(t)}=G\left(H_{0} D^{0}\right) X_{(t-h)^{\prime}} \quad H_{0}=1$

- Definición 5.1. El modelo de función de transferencia de m-ésimo orden, m $=(r, s)$, para procesos que forman sistemas lineales invariantes en tiempos continuos se define como:

$$
\left\{1+C_{1} D+C_{2} D^{2}+\ldots+C_{r} D^{r}\right\} Y_{(t)}=G\left(1+H_{1} D^{1}+\ldots+H_{s} D^{s}\right) X_{(t-b)}
$$

- Definición 5.2. El modelo de función de transferencia de m-ésimo orden $m \geq 1$ para procesos que forman sistemas lineales dinámicos invariantes en el tiempo discreto se define como:

$\left\{1+\zeta_{1} \tilde{N}+\ldots+\zeta_{r} \nabla^{r}\right\}_{t}=g\left(1+h_{1} \tilde{N}+\ldots+h_{s} \nabla^{s}\right) X_{t-b}$ 
- Lema 5.1. La función impulso respuesta es un cociente de funciones del operador de retardo.

Demostración:

Sea $\left\{1+\zeta_{1} \tilde{N}+\ldots+\zeta_{r} \nabla\right\} Y_{t}=g\left(1+h_{1} \tilde{N}+\ldots+h_{s} \nabla^{s}\right) X_{t-b}$

Para $B=(1-\nabla)$ operador de retardo, la ecuación se transforma en:

$\left\{1-\delta_{1} B-\ldots-\delta_{r} B^{r}\right\} Y_{t}=\left(\omega_{0}-\omega_{1} B-\ldots-\omega_{s} B^{s}\right) X_{t-b^{\prime}}$ donde $\delta$ y $\omega$ son ponderaciones.

$d(B) Y_{t}=w(B) X_{t-b}$

$Y_{t}=\frac{\omega(B)}{\delta(B)} X_{t-b}$

Luego I(B) $=\frac{\omega(B)}{\delta(B)}$, función impulso respuesta.

En este ejemplo se explica la importancia de los exponentes $r$ y $s$ en la determinación del modelo:

Ejemplo 1:

Sea $r=2$ y $s=2$

Entonces: $\left\{1-\delta_{1} B-\delta_{2} B^{2}\right\} Y_{t}=\left(\omega_{0}-\omega_{1} B-\omega_{2} B^{2}\right) X_{t-b}$

Pero por el lema anterior $Y_{t}=I(B) X_{t-b}$

Luego: $Y_{t}=\left(I_{0}+I_{1} B+I_{2} B^{2}\right) X_{t-b}$

$Y$ reemplazando (2) en (1), y agrupando:

$I_{0}+\left(I_{1}-I_{0} \delta_{1}\right) B+\left(I_{2}-I_{1} \delta_{1}-I_{0} \delta_{2}\right) B^{2}+\left(-I_{1} \delta_{2}-I_{2} \delta_{1}\right) B^{3}=\omega_{0} B^{b}-\omega_{1} B^{b+1}-\omega_{2} B^{b+2}-\ldots$

donde los elementos del miembro izquierdo de la ecuación son potencias j en $B$ para $\mathrm{j}=0,1,2,3$ y en el miembro derecho de la ecuación los elementos son potencias en $b+\ell$, para b períodos de retardo y $\ell=0,1,2, \ldots$ Igualando y haciendo variar $j$, b y s se obtienen los valores $I_{i}=f\left(d, w_{1}\right)$.

\subsection{Algunos casos particulares}

Para la identificación del modelo bivariado, se hace necesario el uso de las ecuaciones anteriores generalizadas, para lo cual será indispensable conocer los valores de r, s, b. (1) (Box et al. 1994).

Solo con el propósito de aclarar más los conceptos se verá cómo se obtienen las ponderaciones $(\mathrm{W}, \mathrm{j})$. 
Ejemplo 1:

Para $r=1, s=0$

$\left\{1+\zeta_{1} \nabla\right\} Y_{t}=g X_{t-b}$

$Y_{t}+\zeta_{1}\left\{Y_{t}-Y_{t-1}\right\}=g X_{t-b}$

además:

$\left\{1-\delta_{1} B\right\} Y_{t}=\omega_{0} B^{b} X_{t}$

$Y_{t}-\delta_{1} Y_{t-1}=\omega_{0} X_{t-b}$

$\frac{Y_{t}-\delta_{1} Y_{t-1}}{\omega_{0}}=X_{t-b}$

reemplazando (2) en (1):

$Y_{t}+\zeta_{1}\left\{Y_{t}-Y_{t-1}\right\}=g \frac{Y_{t}}{\omega_{0}}-g \frac{\delta_{1} Y_{t-1}}{\omega_{0}}$

$\left(1+\zeta_{1}\right) Y_{t}-\zeta_{1} Y_{t-1}=g \frac{Y_{t}}{\omega_{0}}-g \frac{\delta_{1} Y_{t-1}}{\omega_{0}}$

igualando miembro a miembro: $1+\zeta_{1}=\frac{g}{\omega_{0}} \quad y \quad \zeta_{1}=g \frac{\delta_{1}}{\omega_{0}}$

de los cuales: $\quad \omega_{0}=\frac{g}{\left(1+\zeta_{1}\right)} \quad$ y $\quad \omega_{0}=\left(1-\delta_{1}\right) g$
finalmente:

$\mathrm{I}_{\mathrm{b}}=\omega_{0}=\frac{\mathrm{g}}{\left(1+\zeta_{1}\right)}=\left(1-\delta_{1}\right) \mathrm{g}$

Dando ahora valores a: b, z, g para calcular la salida para cualquier entrada: Sea una unidad de retardo para la entrada $(b=1)$, un coeficiente $\zeta=2$ y una ganancia $g=3$ :

$\left\{1+\zeta_{1} \nabla\right\} Y_{t}=g X_{t-b}$

$\{1+2 \nabla\} Y_{t}=3 X_{t-1}$

O,

$\left\{1-\delta_{1} B\right\} Y_{t}=\omega_{0} X_{t-1}$

luego por el resultado en (a):
$\omega_{0}=\frac{g}{1+\zeta_{1}}=1$
(i.e. $\omega_{0}=1$ )
$\omega_{0}=\left(1-\delta_{1}\right) g$, entonces: 
$1-\frac{\omega_{0}}{\mathrm{~g}}=\delta_{1} \quad$ (i.e. $\left.\delta_{1}=2 / 3\right)$

Luego la ecuación lineal general es: $(1-(2 / 3) B) Y_{t}=X_{t-1}$

Entonces, si se asume un valor inicial para $Y_{0}=0, X_{0}=0$ y $X_{1}=X_{2}=\ldots=1$, los tres primeros valores observados del proceso de salida son:

$\mathrm{Y}_{\mathrm{t}}=(2 / 3) \mathrm{Y}_{\mathrm{t}-1}+\mathrm{X}_{\mathrm{t}-1}$

$Y_{1}=(2 / 3) Y_{0}+X_{0}=0$

$Y_{2}=(2 / 3) Y_{1}+X_{1}=1$

$Y_{3}=(2 / 3) Y_{2}+X_{2}=5 / 3$

Las ecuaciones (a), (b) y (c) son ecuaciones claves para expresar la ecuación diferencial general y se pueden sintetizar estos resultados en la siguiente tabla que presenta las ponderaciones, la función impulso respuesta y la función respuesta de paso para algunos valores de ry de s, obtenidas a partir de las ecuaciones (1) y (2).

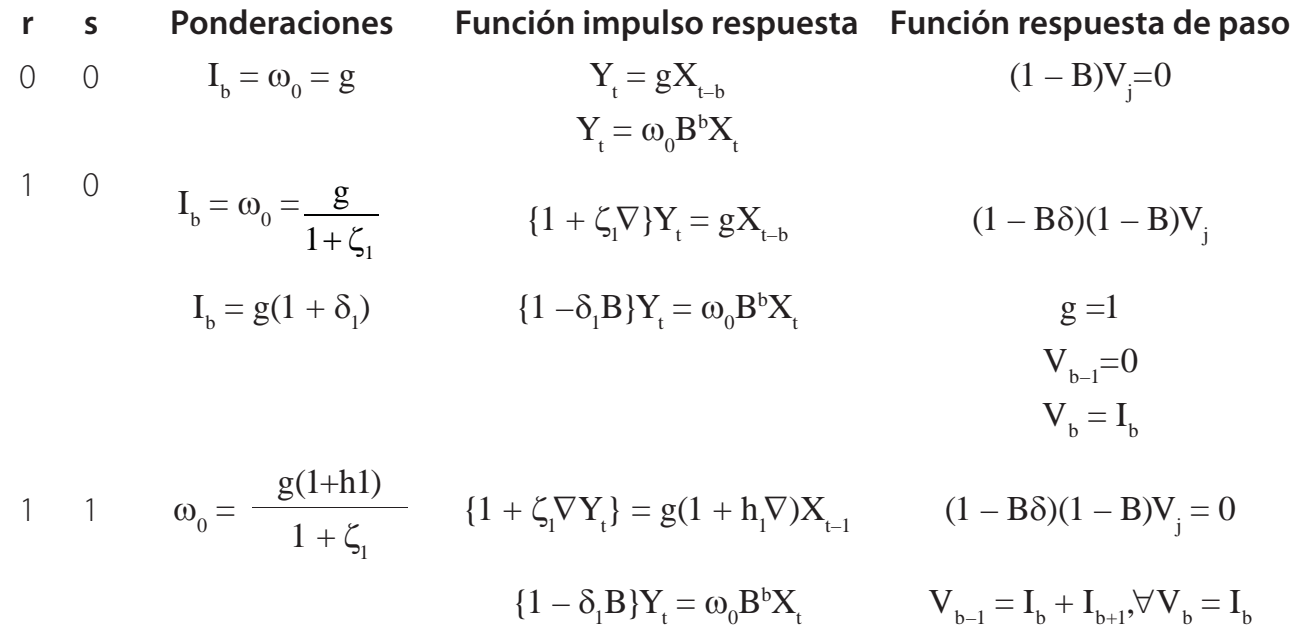

Depende de las raíces $\mathrm{S}_{1}^{-1}, \mathrm{~S}_{2}^{-2}$ de la ecuación característica $\left(1-\delta_{1} \mathrm{~B}-\delta_{2} \mathrm{~B}^{2}\right)=\left(1-\mathrm{S}_{1} \mathrm{~B}\right)$ $\left(1-S_{2} B\right)=0$, donde $\left(1-S_{1} B\right)\left(1-S_{2} B\right)$ es una forma cuadrática.

20 Entonces, analizando el discriminante:

Si $\delta_{1}^{2}+4 \delta_{2}>0$, las raíces de $\mathrm{S}_{1}^{-1}, \mathrm{~S}_{2}^{-2}$ son reales.

Si $\delta_{1}^{2}+4 \delta_{2}<0$, las raíces de $\mathrm{S}_{1}^{-1}, \mathrm{~S}_{2}^{-2}$ son complejas.

Si $\delta_{1}^{2}+4 \delta_{2}=0$, las raíces de $\mathrm{S}_{1}^{-1}, \mathrm{~S}_{2}^{-2}$ son reales e iguales.

Tabla 1. Ponderaciones, función impulso respuesta y función respuesta de paso según valores de r y s Elaboración propia. 


\subsection{Sistemas perturbados}

Antes de concluir con los sistemas lineales y funciones de transferencia, se presentará un factor adicional que interviene en todo sistema, y que se conoce como proceso de perturbación $\{\mathrm{N}\}$.

- Definición 7.1. El sistema lineal dinámico perturbado por la intervención del proceso $\left\{N_{1}\right\}$ en el proceso de salida se define como:

$Y_{t}=I(B) X_{t-b}+N_{t}$

$Y_{t}=d^{-1}(B) w(B) X_{t-b}+N_{t}$

Se asume que $\{N\}$ es un proceso incorrelacionado con el proceso de entrada $\{X\}$. Además, el proceso de perturbación $\{\mathrm{N}\}$ no necesariamente es un ruido blanco, es decir, $\{\mathrm{N}\}$ puede no ser un proceso puramente aleatorio (Brokwell y Davis 2002). Gráficamente:

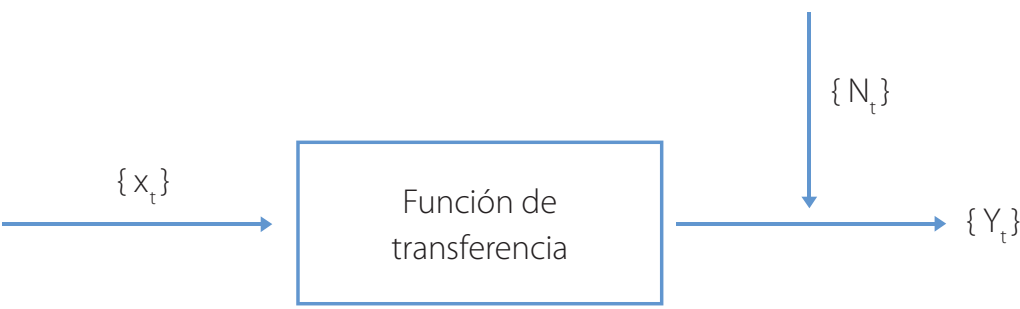

Figura 5. Sistema lineal dinámico perturbado

Elaboración propia.

\subsection{Los procesos ARIMA como sistemas dinámicos}

En la modelización de series de tiempo univariada y bivariada es muy común el uso de los modelos ARIMA para obtener estimaciones a futuro. Partiendo de las condiciones de estacionariedad e invertibilidad se trabajan series con un comportamiento marcado por la tendencia y los ciclos, como también aquellas que incorporan movimientos a corto plazo, es decir estacionalidad (Peña 2010). En tal sentido, se presentan los procesos ARIMA como parte de los sistemas que se vienen analizando.

- Lema 8.1. Los procesos ARIMA son un caso particular de procesos que forman sistemas lineales dinámicos.

Demostración:

Sea un proceso ARIMA: $F(B) Z_{t}=\theta(B) a_{t}$ 
Luego:

$\mathrm{Z}_{\mathrm{t}}=\Phi^{-1}(\mathrm{~B}) \theta(\mathrm{B}) \mathrm{a}_{\mathrm{t}}$

visto de esta forma el proceso de entrada será $\{a\}$ y el proceso de salida $\{Z\}$, la función impulso respuesta sería:

$l(B)=\Phi^{-1}(B) \theta(B)$

Luego $Z_{t}=\mid(B) a_{t}$ que es un modelo de función de transferencia para un sistema lineal dinámico.

Por extensión se pueden incluir los procesos ARIMA estacionales, conocidos COMO SARIMA.

\subsection{Los procesos estables o invertibles}

Los procesos de interés son los procesos estacionarios; si no cumplen con esta condición, la estacionariedad puede ser lograda por diferenciación (Pérez 2006) pero otra condición necesaria para el sistema conformado por los procesos $\{X\}$ y $\{Y\}$ es la condición de estabilidad.

- Definición 9.1. El sistema lineal dinámico se dice que es estable si $\sum_{\mathrm{i}=0}^{\infty} \mathrm{I}_{\mathrm{i}} \mathrm{B}^{\mathrm{i}}$

converge para $|\mathrm{B}| \leq 1$. Esta condición de estabilidad implica que incrementos finitos en la entrada originan incrementos finitos en la salida. En consecuencia, si el sistema es estable se tiene que:

$$
\sum_{i=0}^{\infty} I_{i}=g
$$

quiere decir que para sistemas estables las sumas de las ponderaciones de impulso respuesta convergen a la ganancia del sistema en estado fijo. La condición de estabilidad de los modelos de función de transferencia es análoga a la condición de estacionariedad para modelos ARIMA.

- Lema 9.1. Un modelo de función de transferencia es estable si las raíces de la ecuación característica caen fuera del círculo unitario.

Según este corolario, si el modelo es de primer orden, será estable si:

$-1<\delta_{1}<1$.

Si el modelo es de segundo orden debe cumplirse:

$\delta_{2}+\delta_{1}<1$

$\delta_{2}-\delta_{1}<1$

$-1<\delta_{2}<1$ para ser estable.

Tómese en cuenta que el uso de las raíces fuera del círculo unitario es usado en el análisis de la estacionariedad de las series de tiempo, siendo 
en realidad una condición de la estabilidad del proceso (Medina 2010). En consecuencia, se debe entender que los procesos estacionarios cumplen con tener raíces fuera del círculo unitario (estabilidad) y no al revés.

\subsection{Otros tipos de sistemas}

El tipo de sistema que se está tratando es el sistema lineal dinámico de causa-efecto para un proceso de entrada y uno de salida. Pero este no es el único, por lo que se presentará en forma muy breve otros dos tipos de sistemas, los cuales tienen cada uno características propias. Estos son:

a) Sistema lineal en serie (sistemas en cascada).

b) Sistemas de retroalimentación (feedback).

\section{A. Sistema lineal en serie (o en cascada)}

Como su nombre lo sugiere, este sistema concatena dos o más procesos, donde cada uno de los procesos intermedios cumple la doble función de ser proceso de entrada y salida. La figura 4 presentada anteriormente ilustra esta situación; en ella se aprecia que el proceso de salida $\left\{Y_{t}\right\}$ constituye un proceso de entrada que a su vez genera una segunda salida $\left\{Z_{\mathrm{r}}\right\}$.

Una característica adicional es que la relación existente entre los tres procesos es de tipo causa-efecto. Luego, para este caso se tendrá dos funciones de impulso respuesta y dos procesos de perturbación.

- Lema 10.1. La función de transferencia para los procesos en cascada son del tipo:

$Z_{t}=l(B) X_{t}+N(t)$

Demostración:

Sean $Z_{t}=I_{2}(B) Y_{t}+N_{2}(t) y Y_{t}=I_{1}(B) X_{t}+N_{1}(t)$

dos sistemas lineales en serie.

$Z_{t}=I_{2}(B)\left[I_{1}(B) X_{t}+N_{1}(t)\right]+N_{2}(t)$

$Z_{t}=I_{1}(B) I_{2}(B) X_{t}+I_{2}(B) N_{1}(t)+N_{2}(t)$

Luego:

$Z_{t}=I(B) X_{t}+N()$, donde I(B) es la función impulso respuesta y $N_{(t)}$ es el proceso de perturbación.

Observe que también aquí se utiliza el operador de retardo, dejando abierta la posibilidad para que la variable $X_{\mathrm{t}^{\prime}}$ o ambas $X_{\mathrm{t}}$ e $\mathrm{Y}_{\mathrm{t}^{\prime}}$ expliquen el comportamiento de la variable $Z_{\mathrm{t}}$ en función de su pasado. 


\section{B. Sistema de retroalimentación (feedlback)}

Este sistema se presentará mediante un ejemplo: se considerará un suceso muy común en toda sociedad. Sea $\{X\}$ biomasa de anchoveta en el litoral peruano e $\left\{Y_{t}\right\}$ variable desove de anchoveta en el litoral peruano. Si aumenta el volumen del desove se espera un incremento de la biomasa, la cual al llegar a la adultez podrá a su vez desovar. Si el fenómeno se invierte, es decir si por efectos de aumento en la temperatura superficial del mar peruano $\left\{\mathrm{N}_{1}(\mathrm{t})\right\}$ esto es, frente a la presencia del fenómeno El Niño, o por el volumen de carga microbiana de origen fecal echada al océano $\left\{\mathrm{N}_{2}(\mathrm{t})\right\}$, y siendo esta una especie pelágica, disminuye el número de individuos en edad de procreación, también disminuirá el volumen de desove y volverá a repetirse el ciclo (Yáñez, González y Barbieri 1995). Lo señalado se ilustra en la figura 6.

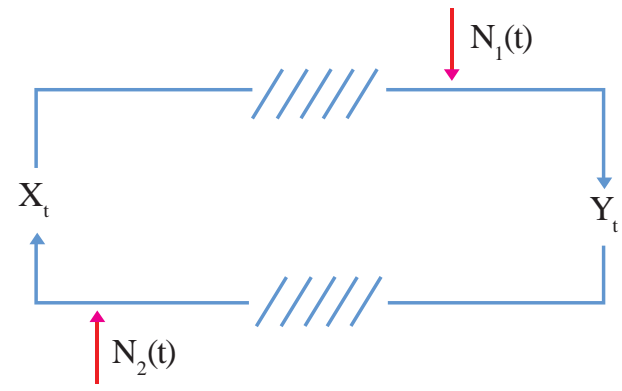

Figura 6. Sistema de retroalimentación o feedback

Elaboración propia.

La variable que en un principio era la de entrada, termina siendo la variable de salida; además, se presentan dos funciones de transferencia y dos procesos de perturbación independientes entre sí. Algo más que caracteriza a este sistema es que la relación en el tiempo no se da en forma puntual, quiere decir que necesariamente debe existir una relación tipo adelanto-retardo para que se cumpla el ciclo.

\section{Aplicación de modelos de series de tiempo SARIMA}

Se modelará un sistema marino conformado por la biomasa de anchoveta, denominada capturas en función de la temperatura del mar peruano, considerando un período desde enero de 1959 hasta diciembre del 2006. Se usarán modelos de series de tiempo SARIMA para presentar en forma univariada los sistemas lineales que conforman ambas series y se culminará presentando una modelización bivariada a manera de ejemplo para los sistemas lineales con perturbación. 


\subsection{Análisis exploratorio de las series temperatura y captura de anchoveta (biomasa)}

Al observar el comportamiento de la componente tendencia de la temperatura de la superficie marina del hemisferio norte, que históricamente se ha recogido desde 1875 por los reportes de la National Oceanic and Atmospheric Administration (NOAA), se aprecia que conforme aumenta el tiempo (meses) la temperatura se eleva en 0,00154 grados centígrados. A partir de 1875 a la fecha han transcurrido aproximadamente 1,644 meses, lo que ha dado lugar a una elevación de 2,53 ${ }^{\circ} \mathrm{C}$; se deja a los especialistas marinos el análisis del efecto de la elevación de la temperatura sobre la vida de las especies pelágicas.

\begin{tabular}{l} 
Fitted Trend Equation \\
$Y \mathrm{t}=26,8351+\odot, 00154^{*} \mathrm{t}$ \\
\hline
\end{tabular}

Mediante un ordenamiento por años, se ha realizado una dócima de hipótesis para la diferencia entre años mediante el método de análisis de varianza para un solo factor, encontrando una diferencia significativa con un p-value cercano a cero.

\begin{tabular}{|lrrrrrr|}
\hline Analysis & of Variance & for & TREN1 & & \\
Source & DF & SS & MS & F & P \\
año & 136 & 8,829976 & 0,064926 & $2,1 E+05$ & $0,00 \odot$ \\
Error & 1503 & 0,000468 & 0,000000 & & \\
Total & 1639 & 8,830444 & & & \\
\hline
\end{tabular}

En series de tiempo es importante contar con un gran número de datos a fin de incorporar las periodicidades, tanto en la exploración como en el modelamiento, especialmente si la serie presenta estacionalidad y ciclos. En las series climáticas, ecológicas o medioambientales, es de especial importancia contar con series no menores a trece años con registros mensuales (Medina 2010, Peña 2010). Por otro lado, en series de tiempo la asociación se mide, en el caso univariado por la autocorrelación (asociación de la serie en diferentes momentos en el tiempo) y en el caso multivariado por la coherencia espectral (Box et al. 1994).

Además, con las pruebas de medias por año con intervalos al 95\% se confirma la existencia de la tendencia hacia el calentamiento global. Sin embargo, en el litoral peruano la tendencia es exactamente contraria a lo que ocurre en el hemisferio norte. Aquí se aprecia una tendencia decreciente con una pendiente -0,00033.

$$
\begin{aligned}
& \text { Fitted Trend Equation } \\
& \mathrm{Yt}=17,1432-0,00 \odot 330^{*} \mathrm{t}
\end{aligned}
$$


Fátima Medina Merino de Aliaga

Trend Analysis Plot for tendenc

Linear Trend Model

$Y t=17.1432-0.000330 * t$

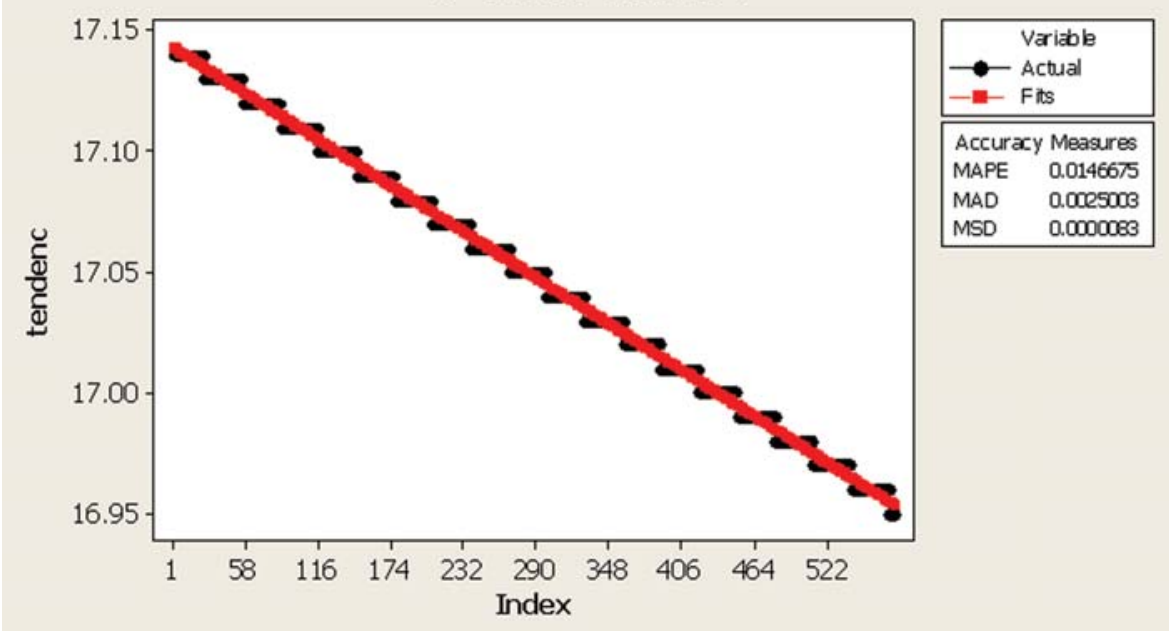

Figura 7. Tendencia de la serie temperatura en el litoral peruano

Elaboración propia.

Un modelo SARIMA para la temperatura superficial del mar peruano expresado en forma implícita será $\mathbf{( 1 , 1 , 0 ) \times ( 1 , 0 , 0 )}$

\begin{tabular}{|llrrrr|}
\hline Type & & Coef & SE Coef & T & P \\
AR & 1 & 0,1877 & $\odot, 0414$ & 4,53 & $\odot, \odot \odot \odot$ \\
SAR & 12 & 0,2198 & $\odot, \odot 417$ & 5,28 & $\odot, \odot \odot \odot$ \\
\hline
\end{tabular}

Con una diferencia en la parte regular. La función de transferencia para este modelo SARIMA será:

$$
\Delta Z_{\mathrm{t}}=(1+0,2052 \mathrm{~B}) /\left(1-0,2189 \mathrm{~B}_{12}\right){ }^{*} \mathrm{a}_{\mathrm{t}}
$$

Los residuos presentan un comportamiento tipo ruido blanco. 


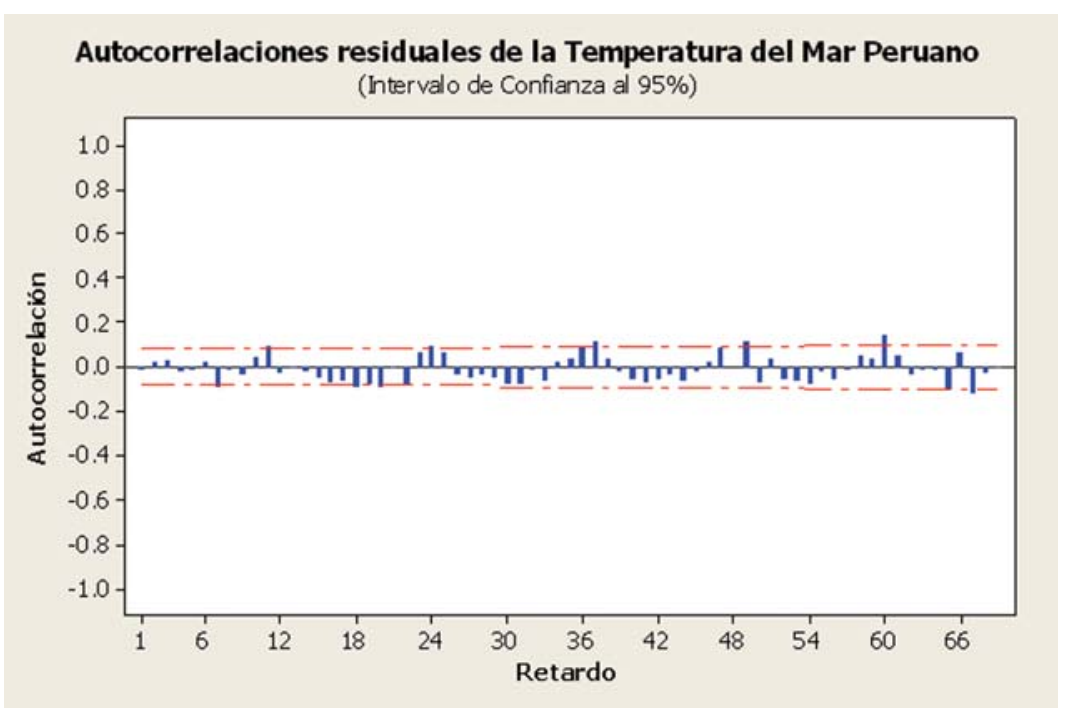

Figura 8. Autocorrelaciones residuales de la temperatura del mar peruano

Elaboración propia.

\begin{tabular}{|c|c|c|c|}
\hline \multicolumn{3}{|c|}{ Ljung-Box Q(18) } & \multirow{2}{*}{ Resultado de la Prueba } \\
\cline { 1 - 2 } Statistics & DF & Sig. & \\
\hline 15,726 & 16 &, 472 & $\begin{array}{c}\text { Los residuales están } \\
\text { incorrelacionados. }\end{array}$ \\
\hline
\end{tabular}

Se puede concluir que el modelo propuesto es adecuado para estimar el desembarque de anchoveta en función de la temperatura superficial del mar peruano.

\subsection{Exploración de la asociación bivariada entre temperatura y biomasa de anchoveta}

Estudios realizados por el Instituto del Mar del Perú (Imarpe) dan cuenta de la preocupación de los científicos marinos (biólogos, oceanógrafos, químicos, físicos, ingenieros) y del sector económico, por las alteraciones que las variaciones en la temperatura superficial del mar ocasionan sobre los principales recursos marinos, dentro de los que destaca la anchoveta. El Imarpe ha desarrollado eventos y publicaciones referentes a este tema y producto de su preocupación se convocaron consultores para la realización de cursos y consultorías sobre el análisis de series de tiempo aplicado a la temperatura superficial del mar y a la biomasa de la anchoveta (Niquen y Bouchon 2004). 
Después de una revisión de los estudios de temperatura superficial del mar del Perú y las investigaciones llevadas a cabo por El Niño Oscilación del Sur (ENOS), se considera muy importante realizar un análisis previo que permita estudiar las características periódicas de la serie y cuantificar su importancia. Para ello se usará la función densidad espectral de la serie temperatura.

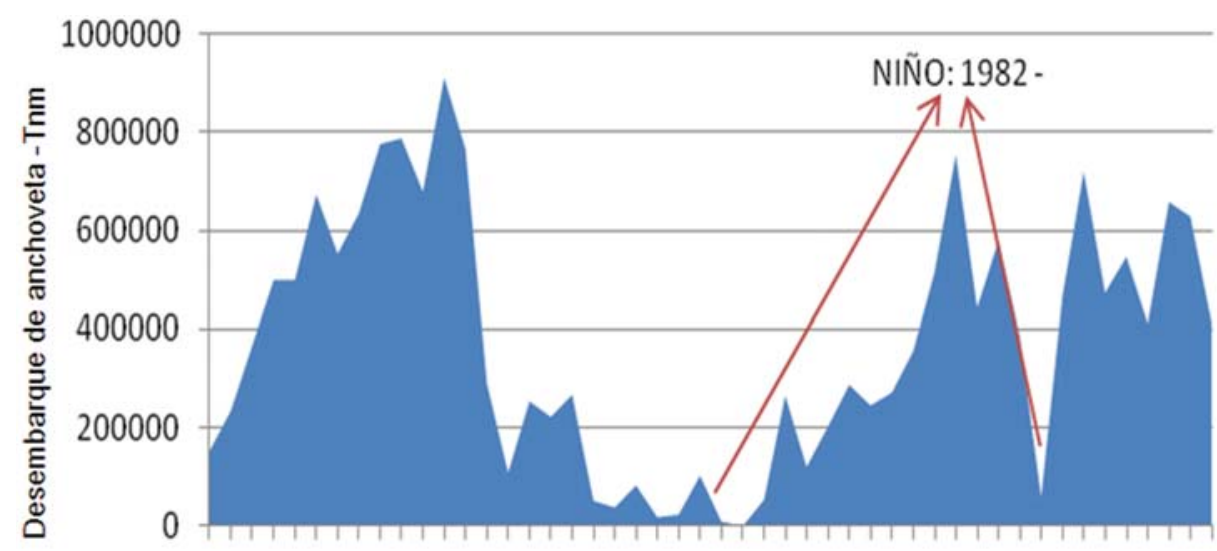

13579911131517192123252729313335373941434547

Figura 9. Total de desembarque de anchoveta, promedio anual. Enero de 1959-diciembre del 2006 Fuente: Imarpe, Dirección de Estudios Pelágicos.

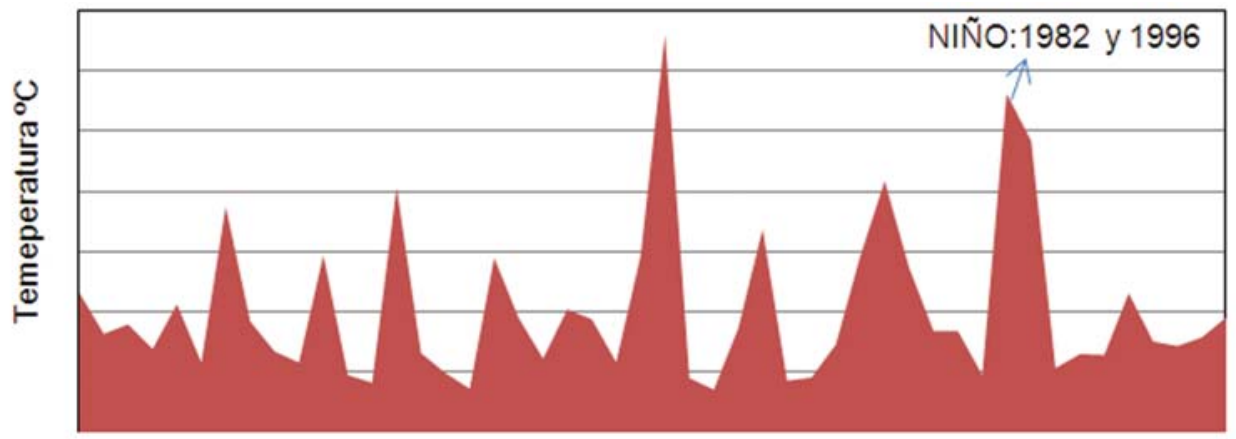

Figura 10. Temperatura superficial del litoral peruano, promedio anual. Enero de 1959-diciembre del 2006

Fuente: Imarpe, Dirección de Estudios Pelágicos. 
El espectro $\mathrm{f}(\mathrm{w})$ es definido como la transformada discreta de Fourier de la función de autocovarianza $\gamma$ de la serie $Y_{t}$.

$$
f(w)=\frac{1}{2 \pi}\left(y_{o}+2 \sum_{j=1}^{\infty} y_{j} \operatorname{Cos}(j w)\right)
$$

Donde $w \hat{I}(0, \pi)$ es la frecuencia angular y $j=1,2,3, \ldots$ corresponde al orden de la autocovarianza. Se usará el espectro con la finalidad de explorar las periodicidades ocultas en la serie temperatura del mar peruano.

Si se considera la captura-desembarque de anchoveta en el litoral peruano como proceso de salida y la temperatura superficial del mar peruano como entrada, la función densidad espectral cruzada es expresada como:

$$
f_{12(w)}=\frac{1}{2 \omega} \sum_{s=-\infty}^{\infty} \rho_{12}(s) e^{-i s w}
$$

"s" debe ser considerado como el desfase en el tiempo entre el proceso de entrada y el de salida. A partir de la función espectral cruzada se analizará la coherencia espectral, a fin de cuantificar la asociación entre la temperatura superficial del mar peruano y la captura-desembarque de anchoveta en el litoral. Sea $\mathrm{f}_{11}(\mathrm{~W})$ la función densidad espectral del proceso de entrada y $\mathrm{f}_{22}(\mathrm{~W})$ la función densidad espectral del proceso de salida, la coherencia espectral $(W(W))$ se determina como:

$$
\mathrm{W}_{12}(\omega)=\frac{\mathrm{f}_{12}(\mathrm{w})}{\left(\mathrm{f}_{11}(\mathrm{w}) \mathrm{f}_{22}(\mathrm{w})\right)^{\frac{1}{2}}}
$$

Y equivale al coeficiente de correlación entre los procesos considerados como entrada-salida.

Propiedades: Para todo $\omega, 0 \leq\left|W_{12}(\omega)\right| \leq 1$.

Si los procesos de entrada y salida que forman el sistema están linealmente relacionados, entonces $W_{12}(\omega)=1$.

El gráfico de la coherencia espectral muestra que existe una asociación muy alta (mayor al 90\%) entre la temperatura superficial del mar peruano y el desembarque de anchoveta para los períodos bajos que corresponde a la estacionalidad y también una asociación importante (aproximadamente 50\%) para los períodos altos (3-4 años) que vienen a ser los ciclos. 
La tabla muestra los resultados de la coherencia y la descomposición espectral cruzada para cada frecuencia (w). Se ha incluido el período al cual corresponden las coherencias más altas.

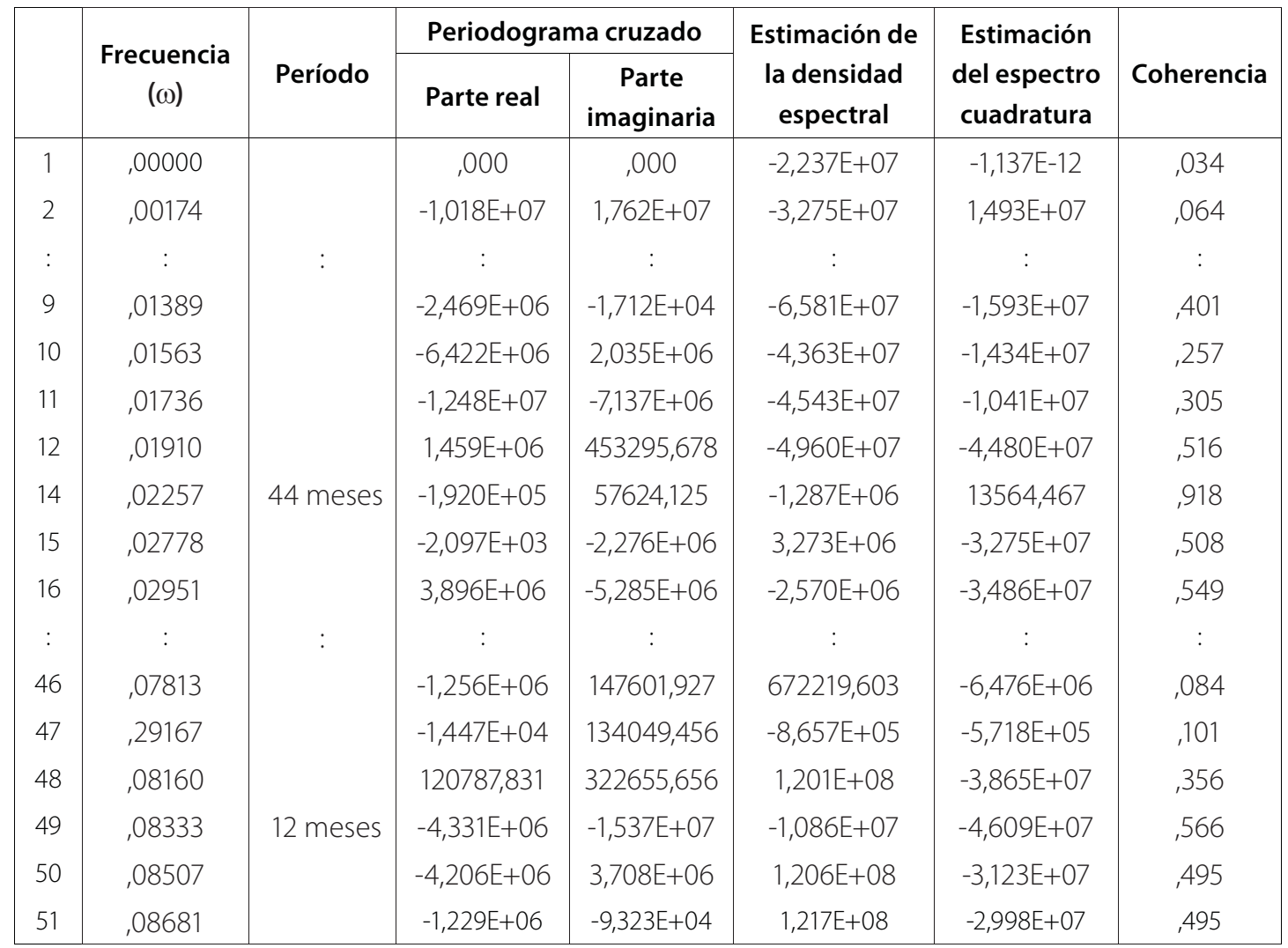

Tabla 2. Descomposición espectral cruzada y coherencia espectral por frecuencias para las series temperatura superficial del mar y desembarque de anchoveta

Elaboración propia. 
Sistemas lineales dinámicos invariantes en series cronológicas

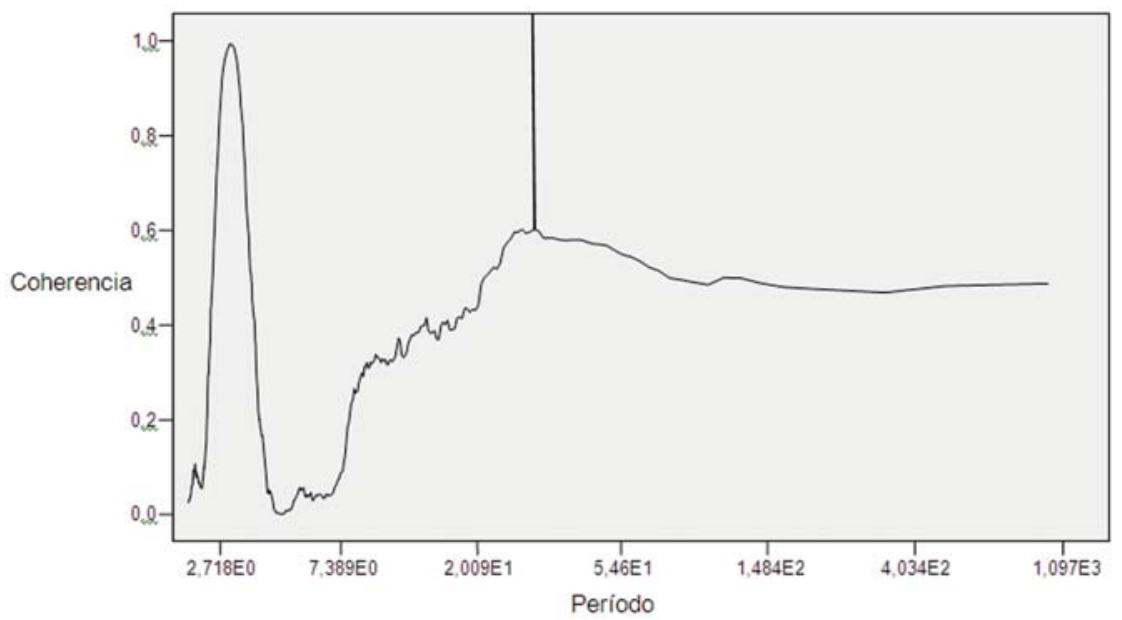

Figura 11. Espectro de coherencia entre temperatura y desembarque de anchoveta Perú: 1959-2006

Elaboración propia.

Finalmente se presenta la función de transferencia del sistema lineal bivariado del tipo causa efecto, cuya identificación se obtiene a partir de la correlación cruzada:

Temperatura promedio anual 1959-2006 con desembarque promedio anual 1959-2006

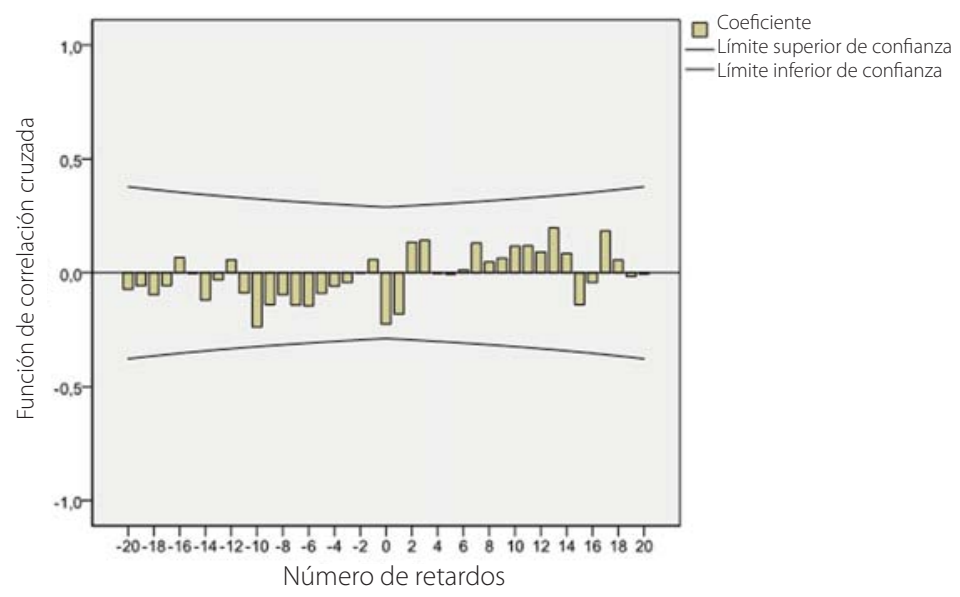

Figura 12. Correlación cruzada: desembarque de anchoveta vs. temperatura del mar peruano

Elaboración propia. 
Fátima Medina Merino de Aliaga

\begin{tabular}{|c|c|c|c|c|c|c|c|}
\hline & & & & Estimación & SE & $\mathrm{t}$ & Sig. \\
\hline $\begin{array}{l}\text { DESEMBARQUE } \\
\text { PROMEDIO ANUAL } \\
1959-2006\end{array}$ & $\begin{array}{l}\text { Sin } \\
\text { transformación }\end{array}$ & $A R$ & Retardo 1 & ,723 & 120 & 6,012 &, 000 \\
\hline \multirow{3}{*}{$\begin{array}{l}\text { TEMPERATURA } \\
\text { PROMEDIO ANUAL } \\
1959-2006\end{array}$} & $\begin{array}{l}\text { Sin } \\
\text { transformación }\end{array}$ & & Diferenciación & 1 & & & \\
\hline & & Numerador & Lag 0 & $-19071,986$ & 9619,199 & $-1,983$ &, 056 \\
\hline & & Numerador, estacional & $\operatorname{Lag} 1$ & 1,874 &, 557 & 3,364 &, 002 \\
\hline
\end{tabular}

Tabla 3. Reporte de modelización de la función de transferencia obtenido con el programa estadístico - SPSS versión 19

Elaboración propia.

La función de transferencia es del tipo $r=0, s=0$ para la parte regular y $r=1, s=0$ para la parte estacional con $d=10$ (retardos)

$$
Y_{t}-0,723 Y_{t-1}=-19071,986 X_{t-10}+1,874 X_{t-22}
$$

\section{Conclusiones}

a) Los modelos ARIMA son una representación de sistema lineal dinámico para procesos univariados de series de tiempo.

b) La entrada del sistema lineal dinámico modelado mediante ARIMA corresponde a procesos desfasados en el tiempo y términos aleatorios, la salida es el proceso mismo bajo estudio.

c) El esquema de parámetros de los procesos modelados mediante ARIMA equivalen a los pesos de la función de transferencia del sistema lineal dinámico.

d) En el caso de los modelos bivariados del tipo causa efecto, se ha mostrado que la relación entre el proceso de entrada y el proceso de salida puede ser modelada por medio de la función de transferencia, incorporando además elementos espectrales para complementar la identificación. 


\section{Bibliografía}

Box, George E. P.; Jenkins, Gwilym y Gregory Reinsel (1994). Time series analysis. Forecasting and control. 3. ${ }^{a}$ edición. Nueva Jersey: Prentice Hall.

Brokwell, Peter y Richard Davis (2002). Introduction to time series and forecasting. 2. edición. Nueva York: Springer.

Medina, Fátima (2010). Análisis de series cronológicas aplicadas a series económicas. Lima: Instituto Nacional de Estadística e Informática, Centro de Investigación y Desarrollo.

Ñiquen, Miguel y Marilú Bouchon (2004). Impact of El Niño events on pelagic fisheries in Peruvian waters, en Milliman, John (ed.). Deepsea research. Part II: Topical studies in oceanography. Oxfordshire: Elsevier Publishing.

Peña, Daniel (2010). Análisis de series temporales. 2. e edición. Madrid: Alianza Editorial.

Pérez, César (2006). Econometría de las series temporales. Madrid: Pearson Prentice Hall/Universidad Complutense de Madrid.

Priestley, Maurice (1981). "Spectral analysis and time series: multivariate series, prediction and control". Spectral analysis and time series. Vol. 2. Londres: Academic Press.

Vidal, Ricardo (1997). Correlación entre las condiciones ambientales y la pesquería de anchoveta a lo largo de la costa de Baja California. México D. F.: Instituto de Investigaciones Oceanológicas/Universidad Autónoma de Baja California.

Yáñez, Eleuterio; González, Antonio y María Barbieri (1995). "Estructura térmica superficial del mar asociada a las distribuciones espacio-temporal de sardina y anchoveta en la zona norte de Chile entre 1987 y 1992". Investigaciones marinas. Vol. 23, pp. 123-147.<http://www.scielo.cl/pdf/imar/v23/art08. pdf>. [Consulta: 10 de julio del 2012]. 